\title{
Does the operator's sex matter? An analysis based on the national interventional cardiology registry
}

\author{
Magdalena Lanocha1,2, Alexandra Lansky³ , Zbigniew Siudak ${ }^{4}$ Adrian Włodarczak², Janusz Tarchalski ${ }^{5}$, \\ Krzysztof P. Malinowski ${ }^{6}$, Witold Laskowski ${ }^{7}$, Tomasz Sikora ${ }^{8}$, Ewa Radziszewska', Magdalena Dolecka-Ślusarczyk ${ }^{4}$, \\ Wojciech Wojakowski ${ }^{9}$, Stanisław Bartuś ${ }^{10}$, Marek Grygier ${ }^{8}$, Tomasz Tokarek $^{10}$, Dariusz Dudek ${ }^{10}$, Maciej Lesiak $^{8}$ \\ 1 St. Adalbert's Hospital, Poznań, Poland \\ 2 Department of Cardiology, Copper Health Center, Lubin, Poland \\ 3 School of Medicine, Yale University, New Haven, Connecticut, United States \\ 4 Faculty of Medicine and Health Science, Jan Kochanowski University, Kielce, Poland \\ 5 Department of Cardiology, Municipal Hospital in Kalisz, Kalisz, Poland \\ 6 2nd Department of Cardiology, Jagiellonian University Medical College, Kraków, Poland \\ 7 Department of Cardiology, Municipal Hospital in Poznań, Poznań, Poland \\ 8 1st Department of Cardiology, Poznan University of Medical Sciences, Poznań, Poland \\ 9 Department of Cardiology and Structural Heart Diseases, Medical University of Silesia, Katowice, Poland \\ 10 Department of Cardiology and Cardiovascular Interventions, University Hospital, Kraków, Poland
}

\section{KEY WORDS}

coronary artery disease, percutaneous coronary intervention, sex outcomes, sex studies

\section{EDITORIAL}

by Zaman, Burgess, and Wing-Lun, see p. 1197
Correspondence to: Magdalena Lanocha, MD, PhD, St. Adalbert's Hospital, ul. B. Krzywoustego 114, 61-144 Poznań, Poland, phone: +48502282996, email: mlanocha@hotmail.com Received: June 30, 2020. Revision accepted: October 15, 2020. Published online: October 22, 2020. Kardiol Pol. 2020; 78 (12): 1221-1226 doi:10.33963/KP.15665 Copyright by the Author(s), 2020

\section{ABSTRACT}

BACKGROUND A small number of female cardiologists work in the field of interventional cardiology. Such disparity is observed in most European countries.

AIMS We present the first national report on the practice patterns and outcomes regarding percutaneous coronary interventions (PCIs) performed by female operators (FOs) in Poland.

METHODS Data were collected from the National Registry of Invasive Cardiology Procedures (Ogólnopolski Rejestr Procedur Kardiologii Inwazyjnej [ORPKI]) between January 2014 and December 2017.

RESULTS A total of $31 \mathrm{FOs}$ (4.1\%) performed 12935 PCIs (2.8\%). The median (interquartile range [IQR]) number of PCIs performed by FOs per year was 75 (43-154), whereas that by male operators was 139 (67-216; $P<0.01)$. Patients handled by FOs were characterized by a lower prevalence of cardiovascular risk factors and previous coronary artery interventions. Acute coronary syndrome was the main indication for treatment (74.66\%). Compared with male operators, FOs handled significantly more patients with single-vessel disease (87.02\% vs 84.72\%; $P<0.001)$. There was no difference in clinical outcomes (a composite of all-cause death, bleeding at the puncture site, or coronary artery perforation) associated with the operator's sex.

CONCLUSIONS Women represent a minority of operators in interventional cardiology and are responsible for a low percentage of PCIs. Nonetheless, the practice patterns and outcomes of PCIs performed by FOs are similar to those of male operators.

INTRODUCTION Statistics are extremely unfavorable for female interventional cardiologists in Poland. Women represent less than $5 \%$ of board-certified interventional cardiologists. Although women constitute $43 \%$ of all cardiologists, only $1 \%$ of them work in the field of interventional cardiology (IC) ${ }^{1,2}$ This small number of female interventional cardiologists contradicts the fact that most medical students are female (75\%). ${ }^{3}$ These observations and disparities can be observed in most European countries and were the rationale for establishing the European Association of Percutaneous Cardiovascular Interventions (EAPCI) Women Committee. One of the aims of the EAPCI is to identify the determinants of these striking sex disparities in the transition from the medical school to IC and to develop strategies for equal career opportunities in the field of IC. ${ }^{4}$

According to an EAPCI Women Committee survey, several barriers preclude women from 


\section{WHAT'S NEW?}

Only $1 \%$ of female cardiologists work in the field of interventional cardiology. This disparity is observed in most European countries. Percutaneous coronary interventions (PCIs) performed by female interventionalists account for less than $3 \%$ of the overall PCI volume in Poland. Female operators (FOs) mostly handle patients with a low prevalence of cardiovascular risk factors. Most FOs are low-volume operators, whereas male operators perform almost double the number of PCIs annually compared with FOs. Nonetheless, our study showed that there was no difference in clinical outcomes, associated with the operator's sex. The practice patterns and outcomes of PCIs performed by FOs are similar to those of male operators. A large multicenter analysis is needed to further investigate the impact of the operator's sex on clinical outcomes in interventional cardiology. Therefore, more FOs should be involved in interventional cardiac procedures.

choosing IC, including lack of opportunity, concerns regarding radiation exposure, and perceived prejudices from male colleagues in the workplace. ${ }^{5}$ Passion is the most common reason for choosing IC. According to male interventional cardiologists responding to the same survey, their most frequently perceived reasons for women not choosing career in IC include on-calls and long working hours in catheterization laboratories.

In contrast, there have been limited data regarding the practice patterns, procedural volumes, and outcomes of percutaneous coronary procedures performed by female interventionalists. Whether the operator's sex impacts the pattern of percutaneous IC procedures remains unknown. Thus, we present the first national report on the practice patterns and outcomes of IC procedures performed by female interventionalists in Poland.

METHODS The National Registry of Invasive Cardiology Procedures (Ogólnopolski Rejestr Procedur Kardiologii Inwazyjnej [ORPKI]) is a nationwide registry of all percutaneous IC procedures performed in Poland. Medical data used in this study were collected from all invasive cardiology sites (catheterization laboratories) between January 2014 and December 2017. Properly anonymized clinical and angiographic characteristics of all IC procedures are electronically submitted to 3 ORPKI modules: angiography only, angiography followed by PCI, and PCI only. Patient informed consent is obtained at the time of original data collection. The registry analysis in this study did not require any ethical approval. Detailed data on the ORPKI registry design have been presented elsewhere. ${ }^{6}$

Statistical analysis Continuous variables were expressed as mean (SD) or median (interquartile range [IQR]) for normally or non-normally distributed variables, respectively. The normality of distribution was assessed using the Kolmogorov-Smirnov and Lilliefors tests. The $t$ test or the Wilcoxon rank sum test were used to compare continuous variables across the 2 study groups. Nominal variables were presented as numbers and percentages, and then compared using the Pearson $X^{2}$ test or the Fisher exact test, as appropriate. The Cochran-Armitage test for trend was used to examine changes over the analyzed years. Pearson or Spearman correlation coefficients were calculated as appropriate. To account for potential selection bias in the comparison of female and male operators, propensity score matching (PSM) was performed using the nearest-neighbor method with the following covariates: age, sex, weight, diabetes, previous stroke, previous myocardial infarction, previous $\mathrm{PCI}$, previous coronary artery bypass grafting, smoking status, psoriasis, hypertension, kidney disease, indication, Killip class, chronic obstructive pulmonary disease, access site, cardiac arrest at baseline, and hypothermia at baseline. The groups were considered balanced if the standardized difference for each of the analyzed baseline or demographic characteristics was lower than $10 \%$. Thrombolysis, the use of glycoprotein IIb / IIIa inhibitors, bivalirudin, fractional flow reserve (FFR), intravascular ultrasound (IVUS), optical coherence tomography (OCT), total radiation dose, total contrast agent dose, aspirin, unfractionated heparins, low-molecular-weight heparins, and complications (bleeding at the puncture site, cardiac arrest, allergic reaction, and death) were regarded as dependent variables in mixed effect models, with each outcome having a separate model. The operator's sex was used as a fixed effect; random effects were used to account for data clustering. A $P$ value less than 0.05 was considered significant. Statistical analysis was performed with the R-3.6.2 software (R Foundation for Statistical Computing, Vienna, Austria).

RESULTS During our study period, 163 Polish catheterization laboratories reported PCI procedures into the ORPKI registry. Of these, only $59(36 \%)$ had at least a single female operator (FO) and 19 (11\%) employed more than one FO (range, 2-5). The ORPKI registry indicated a total of 867 interventional operators performing diagnostic procedures ( 46 women [5.3\%] vs 821 men [94.7\%]) and 757 operators performing diagnostic and therapeutic procedures (31 women [4.1\%] vs 726 men [95.9\%]) over 4 years. The annual trend of the number of FOs performing therapeutic procedures was as follows: 23 (3.6\%) in 2014; 25 (3.7\%) in 2015; 29 (4.1\%) in 2016; $30(4.5 \%)$ in $2017(P=0.44)$.

A total of 1272396 consecutive procedures (447390 angiography only, 368551 angiography followed by PCI, and 456455 PCI only) were included in the analysis. Of the total reported 
TABLE 1 Diagnostic coronary angiography characteristics based on data from the ORPKI registry collected between January 2014 and December 2017

\begin{tabular}{|c|c|c|c|c|}
\hline Parameter & $\begin{array}{l}\text { All operators } \\
(n=867)\end{array}$ & $\begin{array}{l}\text { Female operators } \\
(n=46)\end{array}$ & $\begin{array}{l}\text { Male operators } \\
(n=821)\end{array}$ & $P$ value \\
\hline Coronary angiography & $815941(100)$ & $29721(3.64)$ & $786220(96.36)$ & $<0.001$ \\
\hline \multicolumn{5}{|l|}{ Indication for angiography } \\
\hline STEMI & $94794(11.62)$ & $3224(10.85)$ & $91570(11.65)$ & \multirow[t]{5}{*}{$<0.001$} \\
\hline NSTEMI & $105212(12.89)$ & $3213(10.81)$ & 101999 (12.97) & \\
\hline UA & $258335(31.66)$ & $8163(27.47)$ & $250172(31.82)$ & \\
\hline SA & $314367(38.53)$ & $13044(43.89)$ & $301323(38.33)$ & \\
\hline Other & $43233(5.3)$ & $2077(6.98)$ & $41156(2.5)$ & \\
\hline \multicolumn{5}{|l|}{ Results of angiography } \\
\hline $\begin{array}{l}\text { No evidence of } \\
\text { atherosclerosis }\end{array}$ & $82559(10.13)$ & $4405(14.83)$ & $78154(9.95)$ & \multirow[t]{6}{*}{$<0.001$} \\
\hline No significant stenosis & $210845(25.87)$ & $7116(23.96)$ & $203729(25.94)$ & \\
\hline Single-vessel disease & $211920(26)$ & $7176(24.17)$ & $204744(26.07)$ & \\
\hline LMCA disease & $2644(0.32)$ & $103(0.35)$ & $2541(0.32)$ & \\
\hline Multivessel disease & $248975(30.55)$ & $8871(29.87)$ & $240104(30.58)$ & \\
\hline Multivessel and LMCA disease & $58047(7.12)$ & $2024(6.82)$ & $56023(7.13)$ & \\
\hline
\end{tabular}

Data are presented as number (percentage) of procedures.

Abbreviations: LMCA, left main coronary artery; NSTEMI, non-ST-segment elevation myocardial infarction; SA, stable angina; STEMI, ST-segment elevation myocardial infarction; UA, unstable angina

procedural volume during the analyzed period, FOs performed 29721 diagnostic coronary procedures (3.6\%) and 12935 percutaneous coronary therapeutic procedures (2.8\%) (TABLES1 and 2). Female and male operators performed a median (IQR) number of 495 (147-994) and 853 (3961361) $(P<0.01)$ diagnostic coronary angiographies, respectively, and 305 (186-619) and 562 (283-869) $(P=0.01)$ PCIs, respectively. The median (IQR) number of PCIs performed by FOs per year was 75 (43-154), which was significantly lower than the median number of procedures performed by male operators (MOs) (139 [67216]; $P<0.01)$.

A subanalysis of the data on diagnostic coronary artery procedures is presented in TABLE 1 and Supplementary material, Table S1. Stable angina pectoris was the main indication for angiography for all operators, but it was a more frequent indication for FOs than for MOs $(43.89 \%$ vs $38.33 \%$; $P<0.001)$. No evidence of atherosclerosis during diagnostic angiography was more often diagnosed in the patient population handled by FOs (14.83\% vs $9.95 \% ; P<0.001)$. However, the presence of left main coronary artery disease or multivessel disease detected by diagnostic angiography was positively correlated with the number of PCIs performed by women $(r=0.54, P<0.001$ and $r=0.52, P<0.001$, respectively).

The physiological assessment during angiography was more frequently used by MOs $(2.24 \%$ vs $1.35 \% ; P<0.001)$. The PSM analysis demonstrated that the odds of FFR use during diagnostic angiography by FOs were significantly lower compared with MOs (odds ratio [OR], 0.47; 95\% CI, 0.4-0.55; $P<0.001)$. However, the number of physiological assessments during diagnostic angiography performed by FOs increased annually from 74 (0.94\%) in 2014 to 126 (1.69\%) in 2017 ( $P<0.001)$. The use of invasive imaging techniques among operators during diagnostic coronary angiography confirmed that OCT was preferred by FOs $(0.42 \%$ vs $0.08 \% ; P<0.001)$ compared with IVUS ( $0.39 \%$ vs $0.5 \%$; $P<0.001)$, which was more often performed by MOs.

The access site during angiography did not differ between operators' sexes. During the analyzed years, radial access increased from $62 \%$ of cases to $80 \%(P<0.001)$. The proportion of radial access use increased with growing operators' experience $(r=0.38, P<0.05 ; r=-0.37, P<0.05)$.

A total of 31 female interventionalists (4.1\%) performed 12935 PCIs (2.8\%) during the 4 analyzed years. Acute coronary syndrome was the main indication for treatment (74.66\%) (tABlE 2). Compared with MOs, FOs performed a larger proportion of procedures in patients with ST-segment elevation myocardial infarction $(27.43 \%$ vs $24.48 \%$; $P<0.001)$. Patients handled by FOs were characterized by a lower prevalence of cardiovascular risk factors and previous coronary artery interventions (PCI or 
TABLE 2 Percutaneous coronary interventions based on data from the ORPKI registry collected between January 2014 and December 2017

\begin{tabular}{|c|c|c|c|c|}
\hline Parameters & $\begin{array}{l}\text { All operators } \\
(n=757)\end{array}$ & $\begin{array}{l}\text { Female operators } \\
\text { (n [\%] = } 31[4.1] \text { ) }\end{array}$ & $\begin{array}{l}\text { Male operators } \\
\text { (n [\%] = } 726[95.9] \text { ) }\end{array}$ & $P$ value \\
\hline Procedures (PCI) & $456455(100)$ & $12935(2.8)$ & $443520(97.2)$ & $<0.001$ \\
\hline \multicolumn{5}{|l|}{ Indication } \\
\hline ACS & $451629(72.18)$ & $9562(74.66)$ & $319956(72.91)$ & \multirow[t]{6}{*}{$<0.001$} \\
\hline STEMI & $111782(24.48)$ & $3548(27.43)$ & $108234(24.4)$ & \\
\hline NSTEMI & $87433(19.15)$ & $2411(18.64)$ & 85022 (19.17) & \\
\hline UA & $130303(28.55)$ & $3603(27.85)$ & $126700(28.57)$ & \\
\hline SA & $122111(26.75)$ & $3245(25.09)$ & $118866(26.8)$ & \\
\hline Other & $4826(1.06)$ & $128(0.99)$ & $4698(1.06)$ & \\
\hline \multicolumn{5}{|c|}{ PCI treatment based on lesion data } \\
\hline Single-vessel disease & $387245(84.79)$ & $11256(87.02)$ & $375989(84.72)$ & \multirow[t]{8}{*}{$<0.001$} \\
\hline LMCA disease & $6082(1.33)$ & $130(1.01)$ & $5952(1.34)$ & \\
\hline Multivessel disease & $39973(8.75)$ & $955(7.38)$ & 39018 (8.79) & \\
\hline Multivessel and LMCA disease & $8476(1.86)$ & $162(1.25)$ & $8314(1.87)$ & \\
\hline Other procedures & $14956(3.27)$ & $432(3.34)$ & $14524(3.27)$ & \\
\hline СТO & $10635(2.33)$ & $180(1.39)$ & $10455(2.36)$ & \\
\hline Bifurcation & $21010(4.60)$ & $378(2.92)$ & $20632(4.65)$ & \\
\hline Rotablation & $2159(0.47)$ & $12(0.09)$ & $2147(0.48)$ & \\
\hline Implanted stent & $405307(88.74)$ & $11505(88.94)$ & $393802(88.73)$ & 0.46 \\
\hline \multicolumn{5}{|l|}{ Stent type } \\
\hline BMS & $20218(4.43)$ & $788(6.09)$ & $19430(4.38)$ & \multirow[t]{6}{*}{$<0.001$} \\
\hline DES & $377392(82.63)$ & $10520(81.33)$ & $366872(82.67)$ & \\
\hline BVS & $4397(0.96)$ & $80(0.62)$ & $4317(0.97)$ & \\
\hline Mixed type (BMS, BVS, DES) & $2553(0.56)$ & $102(0.79)$ & $2451(0.56)$ & \\
\hline No stent used & $51425(11.26)$ & $1430(11.06)$ & 49995 (11.27) & \\
\hline$\geq 2$ stents & $71621(15.68)$ & $1865(14.42)$ & $69756(15.72)$ & \\
\hline DEB & $16147(3.54)$ & $472(3.65)$ & $15675(3.54)$ & 0.48 \\
\hline \multicolumn{5}{|c|}{ Periprocedural complications during PCI } \\
\hline Coronary artery perforation & $782(0.17)$ & $22(0.17)$ & $760(0.17)$ & 0.97 \\
\hline Bleeding at the puncture site & $449(0.1)$ & $8(0.06)$ & $441(0.1)$ & 0.18 \\
\hline Death during procedure & $2101(0.46)$ & $74(0.57)$ & $2027(0.46)$ & 0.06 \\
\hline
\end{tabular}

Data are presented as number (percentage) of procedures.

Abbreviations: ACS, acute coronary syndrome; BMS, bare metal stent; BVS, bioresorbable scaffold; CTO, chronic total occlusion; DEB, drug-eluting balloon; DES, drug-eluting stent; LMCA, left main coronary artery; PCI, percutaneous coronary intervention; others, see TABLE 1

coronary artery bypass graft surgery) (Supplementary material, Table S2). Moreover, FOs performed significantly more procedures in patients with single-vessel disease $(87.02 \%$ vs $84.72 \%$; $P<0.001)$ except the left main vessel $(2.26 \%$ vs $3.21 \% ; P<0.001)$ or bypass graft $(0.94 \%$ vs $1.28 \%$, $P<0.001)$. Multivessel disease or complex lesions, such as chronic total occlusion, calcified lesions, and bifurcation lesions, were more frequently observed in patients handled by MOs (TABLE2).
With the increase in female operators' experience, a slight upward trend was observed in their treatment of complex lesions $(r=0.37, P<0.05)$.

In the context of treatment, drug-eluting stents were widely used by all operators (82.63\%), but MOs more often used multiple stents during single procedures than $\mathrm{FOs}$ did $(15.72 \%$ vs $14.42 \%$; $P$ <0.001) (Supplementary material, Table S2). Nevertheless, an increase in the number of stents used per procedure was observed in 
the group of experienced FOs $(r=0.5, P<0.01)$. As shown by the PSM analysis, low-molecular-weight heparins were less commonly used by FOs compared with MOs (OR, 0.6; 95\% CI, 0.44-0.8; $P<0.001)$, but opposite results were noted for unfractionated heparins (OR, 1.19; 95\% CI, 1.11-1.28; $P<0.001)$, and the probability of using glycoprotein IIb / IIIa inhibitors during PCI was significantly higher for FOs than for MOs (OR, 2.34; 95\% CI, 1.59-3.51; $P<0.001$ ). In addition, a moderate correlation could be observed for the use of glycoprotein IIb / IIIa inhibitors by high-volume FOs compared with MOs $(r=0.54$, $P<0.01$ and $r=0.6, P<0.001$, respectively).

Additional imaging techniques (mainly IVUS-guided PCI) were more often used by MOs $(0.81 \%$ vs $0.32 \%$; $P<0.001)$ (Supplementary material, Table S2). However, the PSM analysis did not confirm any statistical significance of IVUS-guided PCIs performed by MOs (OR, 0.574; 95\% CI, 0.32-1.001; $P=0.054$ ).

The PSM analysis confirmed that the total radiation dose used by MOs was significantly lower (by $54.8 \mathrm{mGy}$ ) during PCI $(P<0.001)$. Furthermore, we observed a significant reduction in the amount of contrast agent (mean [SD], from $175[81] \mathrm{cm}^{3}$ to $\left.167[78] \mathrm{cm}^{3} ; P<0.001\right)$ and radiation (median [IQR], from 1013 [502-1779] mGy to 767 [453-1221] mGy; $P$ <0.001) by FOs over the analyzed years.

Regarding patient outcomes (a composite of all-cause death, bleeding at the puncture site, or coronary artery perforation) following diagnostic and therapeutic procedures, there was no difference caused by the operator's sex (TABLE2).

DISCUSSION The ORPKI registry provides a unique insight into the contemporary PCI practice patterns in Poland. The national scope and comprehensive data allowed us to compare the procedural characteristics and outcomes associated with interventionalists' sex.

Data from the ORPKI registry confirmed the staggering disproportion of practicing female interventionalists compared with MOs in Poland. This trend has been observed in numerous countries. In France, women constitute only $3 \%$ of interventional cardiologists ${ }^{7}$; in the United States, only $4.5 \%$ of board-certified interventional cardiologists ${ }^{8}$; in Australia and New Zealand, only $4.8 \%$ of physicians performing interventional procedures. ${ }^{9}$ In Poland, there has been an upward trend (an increase of 0.9 percentage points) in the number of FOs in recent years. However, it has not changed significantly, and this number remains low. As a result, FOs work in a highly male-dominated environment and lack mentorship from female peers within the same catheterization laboratories.

Percutaneous coronary interventions performed by female interventionalists account for less than $3 \%$ of the overall PCI volume in Poland. Similar results were presented by Wang et $\mathrm{al},{ }^{8}$ based on data from the United States $\mathrm{Na}$ tional Cardiovascular Data Registry. Furthermore, our analysis showed that $3.6 \%$ of all diagnostic coronary procedures were performed by FOs, but $2.8 \%$ were elective PCIs; this confirms the fact that women are assigned to less demanding procedures in advance. Insufficient procedural experience may explain the gap between the diagnostic and therapeutic procedure involvement of FOs. In a study by Wang et $\mathrm{al},{ }^{8}$ most FOs were found to be low-volume operators compared with MOs. Nonetheless, Polish FOs are higher-volume operators compared with FOs in the United States; the median (IQR) number of PCIs performed annually by FOs in Poland is 75 (43-154), as opposed to 48 (22-87) in the United States. ${ }^{8}$ As recommended by the Association of Cardiovascular Intervention of the Polish Cardiac Society, each operator should perform a minimum number of $75 \mathrm{PCI}$ procedures annually. Therefore, FOs barely reach this minimum, whereas MOs perform almost double the number every year.

Previous studies have demonstrated that low-volume operators more often perform emergency PCIs compared with high-volume operators, and the patients of the former have fewer cardiovascular comorbidities ${ }^{8,10}$; this trend is in line with our observations. Low-volume FOs are less likely to be given an opportunity to treat more complex lesions such as chronic total occlusion and left main coronary artery or calcific lesions. Therefore, in routine practice, case selection for FOs is mainly focused on stable patients with a lower prevalence of cardiovascular risk factors. We further observed in our analysis that an increase in FOs' experience contributed to only a slight upward trend in complex lesion treatment by women. Thus, additional factors may influence the spectrum of patients treated by FOs.

The use of the physiological assessment (FFR) or additional imaging techniques (IVUS and OCT) is highly recommended by the European Society of Cardiology guidelines on myocardial revascularization. ${ }^{10}$ Our study confirmed that both groups of operators do not widely use OCT, IVUS, or FFR in routine practice, but an upward trend can be noticed during the analyzed years. However, the significantly higher probability of conducting the OCT assessment by FOs during diagnostic procedures should be interpreted with caution due to the small overall number of performed procedures. Furthermore, low-volume operators are more likely to use an additional physiological and imaging assessment during diagnostic procedures. Based on recommendations, FFR-guided $\mathrm{PCI}$ is performed in patients with multivessel disease or left main lesions; these indications predominated for MOs 
in our analysis. In general, the choice of treatment and the combination of additional physiological or imaging assessment techniques depend on each patient's characteristics, comorbidities, and clinical settings. Therefore, the lower involvement of FOs in the treatment of complex lesions and patients with a higher incidence of comorbidities may be responsible for the described discrepancies in patient management.

Finally, the current literature provides contradictory data regarding the relationship of the operator volume with post-PCI outcomes. Some studies have demonstrated that high-volume operators have a significantly lower rate of in-hospital deaths than low-volume operators (OR, $0.79 ; 95 \%$ CI, 0.75-0.83). ${ }^{11}$ In contrast, the last published data from the ORPKI registry showed that there was no significant correlation between operator volume and the procedure-related mortality rate in the overall group of patients undergoing PCI. ${ }^{12}$ Our analysis is consistent with these results; procedure-related mortality did not significantly differ between high-volume male and low-volume female operators.

The increasing proportion of women performing medical professions is a worldwide observation. Hopefully, the presented clinical data and initiatives such as those of the EAPCI Women Committee will support women and bring more highly motivated FOs into catheterization laboratories.

Study limitations Factors affecting sex parity in cardiology are complex and diverse, but they did not represent the subject of our research. Nevertheless, they may significantly influence the current practice pattern in the field of IC. Statistical significance strongly depends on the study sample size. Due to large sample sizes, we observed statistical significance even in terms of small treatment effects (eg, differences in the mean amount of the contrast agent dose of $1.39 \mathrm{ml}$ for angiography and $3.12 \mathrm{ml}$ for PCI between operators), which may remain clinically irrelevant. Finally, ORPKI records are limited to clinical and procedural data without further hospital and long-term follow-up.

Conclusions Data from the ORPKI registry provide a contemporary view of the current IC practice in Poland. Women represent a minority of operators in IC and are responsible for a small percentage of PCIs. The practice patterns of PCI performed by female interventionalists are similar to those of MOs and comply with the European Society of Cardiology guidelines on myocardial revascularization. Future multicenter analysis is needed to further investigate the impact of the operator's sex on clinical outcomes in IC.

\section{ARTICLE INFORMATION}

CONFLICT OF INTEREST None declared.

OPEN ACCESS This is an Open Access article distributed under the terms of the Creative Commons Attribution-NonCommercial-NoDerivatives 4.0 International License (CC BY-NC-ND 4.0), allowing third parties to download articles and share them with others, provided the original work is properly cited, not changed in any way, distributed under the same license, and used for noncommercial purposes only. For commercial use, please contact the journal office at kardiologiapolska@ptkardio.pl.

HOW To CITE Lanocha M, LanskyA, Siudak, et al. Does the operator's sex matter? An analysis based on the national interventional cardiology registry. Kardiol Pol. 2020; 78: 1221-1226. doi:10.33963/KP.15665

\section{REFERENCES}

1 Central Physician Registry [in Polish]. https://old.nil.org.pl/rejestry/centralny-rejestr-lekarzy. Accessed September 15, 2018.

2 Registry of interventional cardiologists at the Association of Cardiovascular Interventions. https://aisn.pl/czlonkostwo/czlonkostwo. Accessed September $15,2018$.

3 Statistics Poland. Higher education institutions and their finances in 2018. https://stat.gov.pl/en/topics/education/education/higher-education-institutionsand-their-finances-in-2018,2,12.html. Accessed December 15, 2019.

4 Buchanan GL, Mehilli J, Kunadian V, et al. The invisible army of women in interventional cardiology: EAPCI Women mission to make them visible. EuroIntervention. 2018; 14: e1158-e1159.

5 Capranzano P, Kunadian V, Mauri J, et al. Motivations for and barriers to choosing an interventional cardiology career path: results from the EAPCI Women Committee worldwide survey. EuroIntervention. 2016; 12: 53-59.

6 Siudak Z, Tokarek T, Dziewierz A, et al. Reduced periprocedural mortality and bleeding rates of radial approach in ST-segment elevation myocardial infarction. Propensity score analysis of data from the ORPKI Polish National Registry. EuroIntervention. 2017; 13: 843-850.

7 Vautrin E, Marlière S, Bellemain-Appaix A, et al. Intervention'elles group Women in interventional cardiology: the French experience. Ann Cardiol Angeiol (Paris). 2016 ; 65: $468-471$.

8 Wang TY, Grines C, Ortega R, et al. Women in interventional cardiology: update in percutaneous coronary intervention practice patterns and outcomes of female operators from the National Cardiovascular Data Registry®. Catheter Cardiovasc Interv. 2016; 87: 663-668.

9 Burgess $S$, Shaw $E$, Ellenberger $K$, et al. Women in medicine: addressing the gender gap in interventional cardiology. J Am Coll Cardiol. 2018; 72: 2663-2667.

10 Neumann FJ, Sousa-Uva M, Ahlsson A, et al. 2018 ESC/EACTS Guidelines on myocardial revascularization. EuroIntervention. 2019; 14: 1435-1534.

11 Fanaroff AC, Zakroysky P, Wojdyla D, et al. Relationship between operator volume and long-term outcomes after percutaneous coronary intervention. Circulation. 2019; 139: 458-472.

12 Zabojszcz M, Januszek R, Siudak Z, et al. Association between the mortality rate and operator volume in patients undergoing emergency or elective percutaneous coronary interventions. Kardiol Pol. 2020; 2: 138-146. 